\title{
THE MODULI SPACE OF COMPLEX LAGRANGIAN SUBMANIFOLDS*
}

\author{
N. J. HITCHIN ${ }^{\dagger}$
}

1. Introduction. Developments in string theory over the past few years (e.g. [13], [5]) have focussed attention on a differential geometric structure induced on the base space of an algebraically completely integrable Hamiltonian system. This has been recently formalised by D.Freed [6] as a special Kähler structure.

The purpose of this paper is to provide an alternative approach to the geometry of special Kähler manifolds, one that is motivated by the desire to understand a more general situation than that afforded by integrable systems. We seek the natural geometrical structure on the moduli space $M$ of deformations of a compact, complex Lagrangian submanifold $Y$ in a complex Kählerian symplectic manifold $X$. In many respects what we do parallels the approach of an earlier paper [8] which began an investigation into the geometry of the moduli space of compact special Lagrangian submanifolds in a Calabi-Yau manifold. This was motivated by a desire to understand the geometry underpinning the Ströminger-Yau-Zaslow [14] approach to mirror symmetry. We are essentially attacking here the special case where the Calabi-Yau is hyperkähler, though we shall not need the full force of the existence of a hyperkähler metric on $X$.

Our viewpoint, as in [8], is to pay less attention to the holomorphic structure of the situation, and more to the symplectic one. Thus a complex Lagrangian submanifold of $X$ can be characterized as a real submanifold on which the real and imaginary parts of the holomorphic symplectic 2 -form vanish. Correspondingly, the differential geometric structure on the moduli space $M$ is induced from a local embedding of $M$ into $H^{1}(Y, \mathbf{R}) \times H^{1}(Y, \mathbf{R})$ which is Lagrangian with respect to two natural constant symplectic forms. We show that this "bilagrangian" condition for a submanifold of a product $V \times V$ of real symplectic vector spaces is equivalent to the structure of a special (pseudo-) Kähler metric on $M$. Moreover, it is easy to see from this point of view that a choice of symplectic basis of $V$ yields the known fact that any special Kähler metric is generated by a single holomorphic function - the holomorphic prepotential.

Finally, we derive from our formalism the hyperkähler metric introduced in a string-theoretic context several years ago by Cecotti, Ferrara and Girardello [2]. It can be seen as a special case of the Legendre transform construction of Lindström and Roček [9], and yields, in the context of our moduli space, a hyperkähler metric on an open set of Markman's moduli space of Lagrangian sheaves [4].

Our approach offers a different perspective to special Kähler geometry, and in particular draws attention to a single naturally defined function $\phi$ which plays an important role: it is the Hamiltonian for the fundamental vector field, a potential for the Kähler metric and, with respect to one of the complex structures, a potential also for the associated hyperkähler metric.

The author wishes to thank Dan Freed for introducing the subject to him and the Institute for Advanced Study for its hospitality.

*Received October 29, 1998; accepted for publication February 11, 1999.

†Mathematical Institute, 24-29 St Giles, Oxford OX1 3LB, England (njh@maths.ox.ac.uk). 
2. Complex Lagrangian submanifolds. Let $X$ be a complex symplectic manifold of complex dimension $2 n$. It has a holomorphic symplectic 2 -form $\omega^{c}$ which we write in terms of its real and imaginary parts:

$$
\omega^{c}=\omega_{1}+i \omega_{2}
$$

These two closed forms are real symplectic forms and define the structure of a complex symplectic manifold on $X$. We see this as follows. Given a closed form $\omega^{c}$, we consider the distribution in $E \subset T \otimes \mathbf{C}$ defined by the complex vector fields $U$ with $\iota(U) \omega^{c}=0$. If $\omega^{c}$ satisfies the algebraic condition that $E \oplus \bar{E}=T \otimes \mathbf{C}$ then it defines an almost complex structure. This is integrable because if $\iota(U) \omega^{c}=0$,

$$
\mathcal{L}_{U} \omega^{c}=d\left(\iota(U) \omega^{c}\right)+\iota(U) d \omega^{c}=0
$$

so if $U$ and $V$ are sections of $E, \mathcal{L}_{U} \omega^{c}=0$ and $\iota(V) \omega^{c}=0$, so

$$
\iota([U, V]) \omega^{c}=\mathcal{L}_{U}\left(\iota(V) \omega^{c}\right)-\iota(V)\left(\mathcal{L}_{U} \omega^{c}\right)=0
$$

and hence $[U, V]$ is a section of $E$.

Similarly we have the following

Proposition 1. A real $2 n$-dimensional submanifold $Y \subset X$ is complex Lagrangian if and only if $\left.\omega^{c}\right|_{Y}=0$.

Proof. If the submanifold $Y$ is complex Lagrangian, then $\left.\omega^{c}\right|_{Y}=0$ by definition. Conversely, if $\left.\omega^{c}\right|_{Y}=0$, we need to show that $Y$ is a complex submanifold, that its tangent spaces are complex. Now the complex structure $I$ on $X$ is defined algebraically by the two real symplectic forms $\omega_{1}, \omega_{2}$. Instead of the description above, we can think of each giving an isomorphism $\varphi_{i}: T X \cong T^{*} X$ and then $I=\varphi_{2}^{-1} \varphi_{1}$. Since $Y$ is Lagrangian with respect to both symplectic forms, then both $\varphi_{1}$ and $\varphi_{2}$ map $T Y$ isomorphically to the conormal bundle $N^{*} Y \subset T^{*} X$. thus $I=\varphi_{2}^{-1} \varphi_{1}$ preserves $T Y$. Hence $Y$ is a complex submanifold, $\left.\omega^{c}\right|_{Y}=0$ and so $Y$ is complex Lagrangian.

We lose nothing therefore by focussing our attention on the symplectic aspects of complex Lagrangian submanifolds. We do, however, need to know that there is a good local moduli space of deformations of $Y \subset X$. In general deformations of compact complex submanifolds can be obstructed, but it follows from the paper of Voisin [15] that this is not the case when the submanifold is Lagrangian. In fact if $X$ has a hyperkähler metric, this is also a consequence of the differential geometric argument of McLean [11]. There thus exists a local moduli space $M$, which is a complex manifold, and such that there is a natural isomorphism

$$
T_{[Y]} M \cong H^{0}(Y, N)
$$

from the tangent space at the point $[Y] \in M$ representing $Y$ and the space of holomorphic sections of the normal bundle $N$ to $Y \subset X$. As $Y$ is complex Lagrangian, $\omega^{c}$ defines a holomorphic isomorphism from $N$ to $T^{*} Y$, so

$$
T_{[Y]} M \cong H^{0}\left(Y, T^{*}\right)
$$

From the holomorphic point of view this is the tangent space to the moduli of deformations of $Y$ as a complex submanifold. The infinitesimal deformations as a complex Lagrangian submanifold correspond to those sections of the normal bundle for which 
the corresponding 1-forms in $H^{0}\left(Y, T^{*}\right)$ are closed. But if we assume that $X$ has a Kähler form $h$ so that $Y$ is also Kähler, then any holomorphic 1-form is closed. Moreover the real dimension of $M$ is then given by $b_{1}(Y)=2 \operatorname{dim}_{\mathbf{C}} H^{0}\left(Y, T^{*}\right)$. We make this Kählerian assumption from now on. We shall investigate next the local differential geometry of $M$.

3. The moduli space. Let $Z$ be a local universal family of deformations of the complex Lagrangian submanifold $Y_{0} \subset X$, so that $Z$ is a complex manifold with a holomorphic projection $\pi: Z \rightarrow M$ and a holomorphic map $F: Z \rightarrow X$ such that

$$
F\left(\pi^{-1}([Y])\right)=Y
$$

Consider the 2 -form $F^{*} \omega_{1}$ on $Z$. If $x_{1}, \ldots, x_{2 n}, y_{1}, \ldots, y_{2 m}$ are real local coordinates on $Z$ with $y_{1}, \ldots, y_{2 m}$ coordinates on $M$ and $\pi\left(x_{1}, \ldots, y_{2 m}\right)=\left(y_{1}, \ldots, y_{2 m}\right)$ then since each fibre $Y$ is Lagrangian with respect to $\omega_{1},\left.F^{*} \omega_{1}\right|_{Y}=0$ and so

$$
F^{*} \omega_{1}=\sum a_{i j} d x_{i} \wedge d y_{j}+\sum b_{i j} d y_{i} \wedge d y_{j}
$$

Furthermore, since $F^{*} \omega_{1}$ is closed,

$$
\sum \frac{\partial a_{i j}}{\partial x_{k}} d x_{k} \wedge d x_{i}=0
$$

We can see in concrete coordinate terms here that, for each $j$, the 1-form $\sum a_{i j} d x_{i}$ on $Y$ is closed. More invariantly, it says that if $U$ is a tangent vector to $M$ at $[Y]$, then if $\tilde{U}$ is a lift to a vector field along $Y$, the 1 -form $\left.\left(\iota(\tilde{U}) F^{*} \omega_{1}\right)\right|_{Y}$ is closed and independent of the choice of lifting.

From (1), integrating $F^{*} \omega_{1}$ over two homologous 1-cycles in a fibre of $\pi$ gives the same result. Now working locally in $M$, we assume that $M$ is contractible, and so we can by homotopy invariance identify the homology of each fibre. Take a homology class $A \in H_{1}(Z, \mathbf{Z}) \cong H_{1}(Y, \mathbf{Z})$ and choose a circle fibration in $\pi: Z \rightarrow M$ such that each fibre is in the class $A$. Integrating along the fibres, we obtain canonically a closed 1 -form

$$
\left(\pi_{A}\right)_{*} F^{*} \omega_{1} \in \Omega^{1}(M)
$$

and since $M$ is assumed contractible, a smooth function $\mu_{A}$ on $M$, well-defined up to an additive constant, such that $d \mu_{A}=\left(\pi_{A}\right)_{*} F^{*} \omega_{1}$. Putting all the functions together gives a map

$$
\mu: M \rightarrow H^{1}(Y, \mathbf{R})
$$

This function by definition has the property that $d \mu(U)$ is the cohomology class of the closed form $\left.\left(\iota(\tilde{U}) F^{*} \omega_{1}\right)\right|_{Y}$.

Proposition 2. $\mu$ is a local diffeomorphism.

Proof. We think in terms of the holomorphic fibration $\pi: Z \rightarrow M$ and a tangent vector $U$ at $[Y]$. The 1-form $\left.\left(\iota(\tilde{U}) F^{*} \omega^{c}\right)\right|_{Y}$ on $Y$ is independent of the choice of lift and thus, taking local holomorphic lifts, is a well-defined global holomorphic 1-form. This is the canonical isomorphism $T_{[Y]} M \cong H^{0}\left(Y, T^{*}\right)$. Now if $d \mu(U)=0$, by the definition of $\mu$, the cohomology class of the real part of $\theta=\left.\left(\iota(\tilde{U}) F^{*} \omega^{c}\right)\right|_{Y}$ is zero. But $Y$ is a Kähler manifold so we have $H^{1}(Y, \mathbf{C}) \cong H^{1,0} \oplus H^{0,1}$ and $\theta+\bar{\theta}$ cannot be 
cohomologically trivial unless $\theta=0$. This means that $\left.\left(\iota(\tilde{U}) F^{*} \omega^{c}\right)\right|_{Y}=0$ and $U=0$. Thus by the inverse function theorem, $\mu$ is a local diffeomorphism.

Similarly, using the other symplectic form $\omega_{2}$, we get a map

$$
\nu: M \rightarrow H^{1}(Y, \mathbf{R})
$$

and, put together, a smooth map

$$
w=(\mu, \nu): M \rightarrow H^{1}(Y, \mathbf{R}) \times H^{1}(Y, \mathbf{R})
$$

Thus $w(M)$ is a smooth submanifold such that the projection onto each factor is a local diffeomorphism.

The vector space $H^{1}(Y, \mathbf{R})$ has a real constant symplectic form defined by the restriction of the Kähler form $h$ on $X$ :

$$
\langle a, b\rangle=\int_{Y} \alpha \wedge \beta \wedge h^{n-1}
$$

where $\alpha, \beta$ are representative 1 -forms for $a$ and $b$. This clearly only requires the cohomology class of the Kähler form $h$. We define two constant symplectic forms on $H^{1}(Y, \mathbf{R}) \times H^{1}(Y, \mathbf{R})$ :

$$
\begin{aligned}
& \Omega_{1}\left(\left(a_{1}, a_{2}\right),\left(b_{1}, b_{2}\right)\right)=\left\langle a_{1}, b_{2}\right\rangle+\left\langle a_{2}, b_{1}\right\rangle \\
& \Omega_{2}\left(\left(a_{1}, a_{2}\right),\left(b_{1}, b_{2}\right)\right)=\left\langle a_{1}, b_{1}\right\rangle-\left\langle a_{2}, b_{2}\right\rangle
\end{aligned}
$$

Take a basis for $V$, so that the skew form has matrix $\omega_{i j}$, then in the corresponding linear coordinates

$$
\begin{aligned}
& \Omega_{1}=2 \sum \omega_{i j} d x_{i} \wedge d y_{j} \\
& \Omega_{2}=\sum \omega_{i j} d x_{i} \wedge d x_{j}-\sum \omega_{i j} d y_{i} \wedge d y_{j}
\end{aligned}
$$

We now have

THEOREM 1. $w(M) \subset H^{1}(Y, \mathbf{R}) \times H^{1}(Y, \mathbf{R})$ is Lagrangian with respect to $\Omega_{1}$ and $\Omega_{2}$.

Proof. The holomorphic symplectic form $\omega^{c}$ is of type $(2,0)$ so since $F$ is holomorphic, $F^{*}\left(\omega^{c}\right)^{2}$ has type $(4,0)$. If $U$ is a tangent vector on $M$ at $[Y]$, then as we have seen, $\left.\left(\iota(\tilde{U}) F^{*} \omega^{c}\right)\right|_{Y}$ is independent of the choice of lifting $\tilde{U}$, because $\left.\omega^{c}\right|_{Y}=0$. Now

$$
\iota(\tilde{U}) \iota(\tilde{V}) F^{*}\left(\omega^{c}\right)^{2}=2\left(\iota(\tilde{U}) \iota(\tilde{V}) F^{*} \omega^{c}\right)\left(F^{*} \omega^{c}\right)+2\left(\iota(\tilde{U}) F^{*} \omega^{c}\right)\left(\iota(\tilde{V}) F^{*} \omega^{c}\right)
$$

and restricting to $Y$,

$$
\left.\iota(\tilde{U}) \iota(\tilde{V}) F^{*}\left(\omega^{c}\right)^{2}\right|_{Y}=\left.\left.2\left(\iota(\tilde{U}) F^{*} \omega^{c}\right)\right|_{Y} \wedge\left(\iota(\tilde{V}) F^{*} \omega^{c}\right)\right|_{Y}
$$

But the left hand side is of type $(2,0)$, hence

$$
\left.\left.\iota(\tilde{U}) \iota(\tilde{V}) F^{*}\left(\omega^{c}\right)^{2}\right|_{Y} \wedge h^{n-1}\right|_{Y}
$$


is of type $(n+1, n-1)$ and so vanishes since $Y$ is complex of dimension $n$. Hence from (6)

$$
\int_{Y} \iota(\tilde{U}) F^{*} \omega^{c} \wedge \iota(\tilde{V}) F^{*} \omega^{c} \wedge h^{n-1}=0
$$

but this means that

$$
\langle(d \mu+i d \nu)(U),(d \mu+i d \nu)(V)\rangle=0
$$

and so equating to zero real and imaginary parts,

$$
\begin{aligned}
& \langle d \mu(U), d \mu(V)\rangle-\langle d \nu(U), d \nu(V)\rangle=0 \\
& \langle d \nu(U), d \mu(V)\rangle+\langle d \mu(U), d \nu(V)\rangle=0
\end{aligned}
$$

These two conditions are precisely the vanishing of $\Omega_{2}$ and $\Omega_{1}$ respectively on $w(M)$.

REMARK. Theorem 1 demonstrates that the structure of the moduli space - defined as a submanifold on which two symplectic forms vanish - parallels the structure of the objects it parametrizes. This is also the philosophy behind the description in [8] of the moduli space of special Lagrangian submanifolds of a Calabi-Yau manifold.

4. Special Kähler manifolds. We shall show that, as a consequence of the "bilagrangian" property of Theorem 1, $M$ inherits a special Kähler structure. The definition, as given in [6], is the following:

Definition 1. A special Kähler manifold is a complex manifold $M$ with

- a Kähler metric $g$ with Kähler form $\omega$

- a flat torsion-free connection $\nabla$ such that

- $\nabla \omega=0$ and

- $d_{\nabla} I=0 \in \Omega^{2}(M, T)$

To clarify the last property, we think of the complex structure $I$, an endomorphism of the tangent bundle $T$, as a 1 -form with values in $T$, i.e. $I \in \Omega^{1}(M, T)$. The connection $\nabla$ defines a covariant exterior derivative $d_{\nabla}: \Omega^{p}(M, T) \rightarrow \Omega^{p+1}(M, T)$ and we require $d_{\nabla} I=0$. This is weaker than $\nabla I=0$ and indeed, since $\omega$ and $I$ determine $g$, the latter condition would say that $\nabla$ is the Levi-Civita connection, and all we would be looking at is a flat Kähler manifold. The reader should be warned that special Kähler manifolds do not form a very interesting class of global Riemannian structures - it has been shown by $\mathrm{Lu}$ [10] that any complete special Kähler manifold is flat.

Now let $V$ be a real symplectic vector space with skew form $\langle$,$\rangle . As in (2),(3)$ we define two constant symplectic forms $\Omega_{1}, \Omega_{2}$ on $V \times V$. We can also define an indefinite metric $g$ on $V \times V$ by

$$
g((a, b),(a, b))=\frac{1}{2}\langle a, b\rangle
$$

Then we have the following

Theorem 2. Let $M \subset V \times V$ be a submanifold which is Lagrangian for $\Omega_{1}$ and $\Omega_{2}$, and transversal to the two projections onto $V$. Then $\left.g\right|_{M}$ is a special pseudoKähler metric. Conversely, any special pseudo-Kähler metric on a manifold $M$ is locally induced from an embedding in $V \times V$ for some $V$. 
Proof. It will be convenient to use the nondegenerate form $\langle$,$\rangle on V$ to identify $V$ with $V^{*}$. Under this identification, $\Omega_{1}$ becomes essentially the canonical symplectic form on $T^{*} V \cong V \times V^{*}$, by setting $d \xi_{i}=\sum \omega_{i j} d y_{j}$ in (4),(5). We then have the following expressions for $\Omega_{1}$ and $\Omega_{2}$ :

$$
\begin{aligned}
& \Omega_{1}=2 \sum d x_{i} \wedge d \xi_{i} \\
& \Omega_{2}=\sum \omega_{i j} d x_{i} \wedge d x_{j}+\sum \omega^{i j} d \xi_{i} \wedge d \xi_{j}
\end{aligned}
$$

To begin, we use the projection onto the first factor $V$ to locally identify $M$ with a flat symplectic vector space. This provides us with our flat connection $\nabla$ with $\nabla \omega=0$. If we use the coordinates $x_{1}, \ldots, x_{2 n}$, then covariant derivatives using $\nabla$ are just ordinary derivatives.

Now since $M$ is Lagrangian with respect to the canonical symplectic form $\Omega_{1}$ on $T^{*} V$ and transversal to the projection to $V$, the embedding is defined by the graph of the derivative of a function on $V$ so in coordinates

$$
\xi_{j}=\frac{\partial \phi}{\partial x_{j}}
$$

for some function $\phi\left(x_{1}, \ldots, x_{2 n}\right)$. The tangent vector $\partial / \partial x_{j}$ of $M$ then lies in $V \times V^{*}$ as the vector

$$
X_{j}=\frac{\partial}{\partial x_{j}}+\sum \frac{\partial \xi_{k}}{\partial x_{j}} \frac{\partial}{\partial \xi_{k}}=\frac{\partial}{\partial x_{j}}+\sum_{k} \frac{\partial^{2} \phi}{\partial x_{k} \partial x_{j}} \frac{\partial}{\partial \xi_{k}}
$$

Since the metric on $V \times V^{*}$ is defined by

$$
g((x, \xi),(x, \xi))=\frac{1}{2}\langle x, \xi\rangle
$$

the induced metric on $M$ is

$$
\sum g_{k j} d x_{k} d x_{j}=\sum g\left(X_{k}, X_{j}\right) d x_{k} d x_{j}=\sum \frac{\partial^{2} \phi}{\partial x_{k} \partial x_{j}} d x_{k} d x_{j}
$$

In general this metric may not be positive definite. It is nondegenerate however, for if $\sum g_{i j} a_{j}=0$, then $\sum a_{j} X_{j}=\sum a_{j} \partial / \partial x_{j}$ so that projection of this tangent vector onto the second factor in $V \times V$ is zero. By the transversality assumption this means each $a_{j}=0$.

Consider now the second Lagrangian condition: $\Omega_{2}$ vanishes on $M$. From (8) this says, using $\xi_{k}=\partial \phi / \partial x_{k}$ and $g_{k j}=\partial^{2} \phi / \partial x_{k} \partial x_{j}$,

$$
0=\Omega_{2}\left(X_{k}, X_{j}\right)=\omega_{k j}+\sum g_{k a} g_{j b} \omega^{a b}
$$

or, writing $I_{k}^{j}=\sum \omega^{j a} g_{a k}$, that $I^{2}=-1$. This is the almost complex structure. Since $\omega_{k j}=-\omega_{j k}, I$ is skew adjoint with respect to $g$. Now let $X$ be the Hamiltonian vector field for the function $\phi$. We have

$$
X=\sum a_{j} \frac{\partial}{\partial x_{j}}=\sum \omega^{i j} \frac{\partial \phi}{\partial x_{i}} \frac{\partial}{\partial x_{j}}
$$


So

$$
\frac{\partial a_{j}}{\partial x_{k}}=\sum \omega^{i j} g_{i k}=I_{k}^{j}
$$

Hence $I=d_{\nabla} X$ and so $d_{\nabla} I=d_{\nabla}^{2} X=0$ since the connection is flat. Hence $I$ satisfies the compatibility condition with the flat connection.

It remains to show that $I$ is integrable. But consider the complex functions

$$
z_{j}=x_{j}-i \sum \omega^{j k} \frac{\partial \phi}{\partial x_{k}}
$$

Differentiating, we obtain the $2 n$ complex 1 -forms

$$
d z_{j}=d x_{j}-i \sum \omega^{j k} \frac{\partial^{2} \phi}{\partial x_{k} \partial x_{l}} d x_{l}=d x_{j}-i \sum \omega^{j k} g_{k l} d x_{l}=d x_{j}-i \sum I_{l}^{j} d x_{l}
$$

and these are clearly of type $(1,0)$. We need to find $n$ linearly independent ones. Let $E \subseteq \Lambda^{1,0}$ be the distribution spanned by $d z_{1}, \ldots, d z_{2 n}$. Then for each $j, 2 d x_{j}=$ $d z_{j}+d \bar{z}_{j}$, and since $d x_{1}, \ldots, d x_{2 n}$ forms a basis, $E \oplus \bar{E}=\Lambda^{1}$ and the rank of $E$ is $n$.

Thus the metric $g$ and the connection $\nabla$ satisfy all the conditions for a special pseudo-Kähler (i.e. possibly indefinite) metric.

Now consider the converse. Let $M$ be a special pseudo-Kähler manifold, and $\left(x_{1}, \ldots, x_{2 n}\right)$ flat local coordinates, so that the covariant derivative is the ordinary derivative and the coefficients of the symplectic form are constant. Consider the 1 -form

$$
\alpha_{k}=\sum \omega_{k l} I_{j}^{l} d x_{j}
$$

Now

$$
d \alpha_{k}=\sum \omega_{k l} \frac{\partial I_{j}^{l}}{\partial x_{m}} d x_{m} \wedge d x_{j}=0
$$

since $\omega_{k l}$ is constant and $d_{\nabla} I=0$. Thus locally there are functions $\xi_{k}$ such that

$$
\alpha_{k}=d \xi_{k} .
$$

We map $M$ to $\mathbf{R}^{2 n} \times \mathbf{R}^{2 n}$ by

$$
\left(x_{1}, \ldots, x_{2 n}\right) \mapsto\left(x_{1}, \ldots, x_{2 n}, \xi_{1}, \ldots, \xi_{2 n}\right)
$$

First we claim the image is Lagrangian with respect to the symplectic form $\Omega_{1}=$ $2 \sum d x_{j} \wedge d \xi_{j}$. But restricting $\sum d x_{j} \wedge d \xi_{j}$ gives

$$
d x_{j} \wedge \alpha_{j}=\sum d x_{j} \wedge \omega_{j l} I_{k}^{l} d x_{k}=\sum d x_{j} \wedge g_{j k} d x_{k}=0
$$

since the metric tensor $g_{j k}$ is symmetric.

Next consider the symplectic form $\Omega_{2}=\sum \omega_{i j} d x_{i} \wedge d x_{j}+\omega^{i j} d \xi_{i} \wedge d \xi_{j}$. Restricted to $M$ this is

$$
\sum \omega_{i j} d x_{i} \wedge d x_{j}+\omega^{a b} g_{a i} d x_{i} \wedge g_{b j} d x_{j}
$$


But since $I^{2}=-1$ this too is zero.

Hence taking $V$ to be $\mathbf{R}^{2 n}$ with the skew form $\omega_{i j}$, we obtain a local embedding of $M$ in $V \times V$, Lagrangian with respect to both forms. It is straightforward to see that the induced metric is $g_{i j}$.

One of the features of the above approach is the fundamental role of the function $\phi$. Here is another aspect of this:

Proposition 3. The function $\phi$ is a Kähler potential.

Proof. Consider

$$
d(I d \phi)=\sum \frac{\partial}{\partial x_{k}}\left(I_{j}^{i} \frac{\partial \phi}{\partial x_{i}}\right) d x_{k} \wedge d x_{j}
$$

Now

$$
\frac{\partial}{\partial x_{k}} I_{j}^{i}=\sum \frac{\partial}{\partial x_{k}}\left(\omega^{i a} g_{a j}\right)=\sum \omega^{i a} \frac{\partial^{3} \phi}{\partial x_{a} \partial x_{j} \partial x_{k}}
$$

so

$$
d(I d \phi)=\sum \omega^{i a} \frac{\partial^{3} \phi}{\partial x_{a} \partial x_{j} \partial x_{k}} \frac{\partial \phi}{\partial x_{i}} d x_{k} \wedge d x_{j}+I_{j}^{i} \frac{\partial^{2} \phi}{\partial x_{i} \partial x_{k}} d x_{k} \wedge d x_{j}
$$

The first term vanishes by the symmetry in $j, k$ and the second term is

$$
\sum I_{j}^{i} g_{i k} d x_{k} \wedge d x_{j}=-\sum \omega_{k j} d x_{k} \wedge d x_{j}=-\omega
$$

where $\omega$ is the Kähler form.

We can now prove

THEOREM 3. The moduli space $M$ of deformations of a complex Lagrangian submanifold of a complex Kählerian symplectic manifold $X$ has a naturally induced special Kähler structure.

Proof. We have to show firstly that $w(M) \subset H^{1}(Y, \mathbf{R}) \times H^{1}(Y, \mathbf{R})$ satisfies the transversality of the previous theorem, but this follows from Proposition 2 applied to $\mu$ and $\nu$. We also need the metric to be definite. But

$$
\begin{gathered}
g(X, X)=\langle d \mu(X), d \nu(X)\rangle=-\frac{i}{2}\langle(d \mu+i d \nu)(X),(d \mu-i d \nu)(X)\rangle \\
=-\frac{i}{2} \int_{Y} \iota(X) \omega^{c} \wedge \iota(X) \bar{\omega}^{c} \wedge h^{n-1}
\end{gathered}
$$

which is definite. We should also show that the complex structure $I$ coincides with the natural complex structure of the moduli space when considered as a moduli space of complex submanifolds. From (9), the functions

$$
z_{j}=x_{j}-i \sum \omega^{j k} \frac{\partial \phi}{\partial x_{k}}
$$


are antiholomorphic with respect to $I$. But $\xi_{j}=\partial \phi / \partial x_{j}$, so $z_{j}=x_{j}-i \sum \omega^{j k} \xi_{k}$. If we return from $V \times V^{*}$ to $V \times V$ using $\omega$ we see that this function is obtained by taking a class $A_{j} \in H_{1}(Y, \mathbf{R})$ and forming

$$
z_{j}=\left\langle\mu-i \nu, A_{j}\right\rangle
$$

Since $d(\mu+i \nu)(U)=\iota(\tilde{U}) \omega^{c}$ is of type $(1,0), d z_{j}=\left\langle d \mu-i d \nu, A_{j}\right\rangle$ is of type $(0,1)$ with respect to the complex structure of the moduli space of compact complex submanifolds, so the two complex structures coincide.

Finally we should pass from the local to the global point of view on $M$, a point which is relevant in particular to the situation where $M$ is the base space of a completely integrable system. There is an additive ambiguity in the choice of the function $\mu$ but $d \mu$ gives an isomorphism between $T M$ and the trivial bundle $M \times H^{1}(Y, \mathbf{R})$, and this is the flat connection $\nabla$ of the special Kähler structure. Globally on $M$, the cohomology of the fibres of $Z \rightarrow M$ defines a flat vector bundle on $M$ (homotopy invariance of cohomology defines the Gauss-Manin connection) and so the isomorphism $d \mu$ provides a flat connection on $T M$. The symplectic form is preserved by the Gauss-Manin connection, and since the complex structure on $M$ is globally defined, so is the metric $g$, which is defined by $I$ and $\omega$.

\section{REMARKS.}

1. One of the well-known features of special Kähler geometry is the fact that any special Kähler metric is derived from a single holomorphic function $\mathcal{F}$ of $n$ variables. It is known as the holomorphic prepotential on $M$. This fact is rather easily seen using our bilagrangian formulism. For this purpose we choose a symplectic basis on $V$. The corresponding coordinates $x_{1}, \ldots, x_{2 n}$ give

$$
\omega=\sum d x_{j} \wedge d x_{n+j}
$$

and so

$$
\begin{aligned}
& \Omega_{1}=2 \sum_{1}^{2 n} d x_{j} \wedge d \xi_{j} \\
& \Omega_{2}=\sum_{1}^{n} d x_{j} \wedge d x_{n+j}-\sum_{1}^{n} d \xi_{j} \wedge d \xi_{n+j}
\end{aligned}
$$

and then

$$
\Omega^{c}=\frac{1}{2} \Omega_{1}+i \Omega_{2}=\sum_{i}^{n} d\left(x_{j}+i \xi_{n+j}\right) \wedge d\left(\xi_{j}+i x_{n+j}\right)=\sum_{1}^{n} d v_{j} \wedge d w_{j}
$$

We see that $\Omega^{c}$ can be identified with the canonical complex symplectic form on $T^{*} \mathbf{C}^{n}$. From Proposition 1, a submanifold on which $\Omega_{1}$ and $\Omega_{2}$ vanish is the same thing as a complex Lagrangian submanifold of $T^{*} \mathbf{C}^{n}$, but this is given by the graph of the derivative of a holomorphic function

$$
v_{j}=\frac{\partial \mathcal{F}}{\partial w_{j}}
$$

From Theorem 2 this is all we need for a special Kähler manifold. 
2. In the standard presentation of the prepotential, its second derivative gives a holomorphic map from the base space of an integrable system to the Siegel upper half-space - the moduli space of polarized abelian varieties, expressed as symmetric matrices with positive definite imaginary part. Such a description involves choosing a symplectic basis for $H_{1}(Y, \mathbf{Z})$ (the classical $A$ and $B$ cycles) which is what we have done to introduce the holomorphic function $\mathcal{F}$. By contrast the real function $\phi$ requires no such choice. All we have chosen is projection onto the first factor in $V \times V$ to define $\phi$. We postpone the discussion of the relationship between $\phi$ and $\mathcal{F}$ to the next section, where we study some associated hyperkähler constructions.

3. In the bilagrangian picture of $M \subset V \times V$ we get another flat torsion-free connection by projecting onto the second factor. From the second Lagrangian condition

$$
0=\left.\Omega_{2}\right|_{M}=\sum \omega_{i j} d x_{i} \wedge d x_{j}-\sum \omega_{i j} d y_{i} \wedge d y_{j}
$$

the pull back of the flat symplectic form under this projection is the same $\omega$. The function $\phi$ is then replaced by its Legendre transform and the new flat coordinates $d y_{j}$ are related to the old ones by $d y_{j}=I d x_{j}$. From the point of view of the moduli space of complex Lagrangian submanifolds, all we have done here is to replace $\omega_{1}$ by $\omega_{2}$, or what is essentially the same, to replace the complex symplectic form $\omega^{c}$ by $i \omega^{c}$. Clearly we can multiply $\omega^{c}$ by $e^{i \theta}$ and still have the same moduli space but now a family of flat connections parametrized by the circle. This is one viewpoint to the study of Higgs bundles as in Simpson's higher dimensional approach [12]. In fact any special Kähler manifold can be thought of as having a Higgs bundle structure on $T \oplus T^{*}$. Recall that a Higgs bundle corresponding to a local system on a Kähler manifold $M$ consists of a holomorphic vector bundle $E$ with a unitary connection $A$ and a section $\Phi \in H^{0}\left(M\right.$, End $\left.E \otimes T^{*}\right)$, the Higgs field, such that the connection

$$
d_{A}+e^{i \theta} \Phi+e^{-i \theta} \Phi^{*}
$$

is flat for all $\theta$. In the case of a special Kähler manifold, the connection $A$ is the Levi-Civita connection on $T \oplus T^{*}$ and the Higgs field has the form

$$
\left(\begin{array}{ll}
0 & 0 \\
\Theta & 0
\end{array}\right)
$$

where $\Theta \in H^{0}\left(M, S^{3} m^{3} T^{*}\right)$ is a holomorphic cubic form. Since Simpson's original approach to Higgs bundles was derived from variations of Hodge structure, this begins to take us back to the picture of a moduli space of complex manifolds which was the original motivation for this paper.

5. Hyperkähler metrics. In [2] Cecotti, Ferrara and Girardello showed how to define naturally a hyperkähler metric on a certain bundle over a special Kähler manifold (see also [6]). As we have seen a special Kähler metric can be defined via a single holomorphic function, so we have a straightforward way of constructing hyperkähler metrics. We shall show here that this construction is in fact a special case of an earlier technique called the Legendre transform construction of Lindström and Roček [9], [7].

Recall (see for example [1]) that a hyperkähler metric is determined by three symplectic forms $\omega_{1}, \omega_{2}, \omega_{3}$ satisfying some algebraic conditions, namely that if $\varphi_{i}$ : $T \rightarrow T^{*}$ is the isomorphism determined by $\omega_{i}$, and we define $J_{1}=\varphi_{3}^{-1} \varphi_{2}, J_{2}=$ $\varphi_{1}^{-1} \varphi_{3}, J_{3}=\varphi_{2}^{-1} \varphi_{1}$, then $J_{1}, J_{2}, J_{3}$ obey the quaternionic identities $J_{1}^{2}=J_{2}^{2}=J_{3}^{2}=$ 
-1 . We work locally first and let $M$ be a special Kähler manifold. On the product $M \times \mathbf{R}^{2 k}$ take the three symplectic 2 -forms $\omega_{1}, \omega_{2}, \omega_{3}$ defined by

$$
\begin{gathered}
\omega_{1}=\sum \frac{\partial^{2} \phi}{\partial x_{j} \partial x_{k}} d x_{j} \wedge d y_{k} \\
\omega_{2}+i \omega_{3}=-\frac{1}{2} \sum \omega_{j k} d\left(x_{j}+i y_{j}\right) \wedge d\left(x_{k}+i y_{k}\right)
\end{gathered}
$$

Then

$$
J_{3}\left(\frac{\partial}{\partial x_{i}}\right)=\sum \omega^{j k} g_{i k} \frac{\partial}{\partial y_{j}}=\sum I_{i}^{j} \frac{\partial}{\partial y_{j}}
$$

and

$$
J_{3}\left(\frac{\partial}{\partial y_{i}}\right)=\sum \omega^{j k} g_{i k} \frac{\partial}{\partial x_{j}}=\sum I_{i}^{j} \frac{\partial}{\partial x_{j}}
$$

So in block matrix form

$$
J_{3}=\left(\begin{array}{ll}
0 & I \\
I & 0
\end{array}\right)
$$

Similarly

$$
J_{2}=\left(\begin{array}{cc}
-I & 0 \\
0 & I
\end{array}\right)
$$

so that $J_{3}^{2}=J_{2}^{2}=-1$ and $J_{2} J_{3}=-J_{3} J_{2}$. Thus $J_{2}, J_{3}$ generate an action of the quaternions, with $J_{1}=J_{2} J_{3}$ given by

$$
J_{1}=\left(\begin{array}{cc}
0 & 1 \\
-1 & 0
\end{array}\right)
$$

and so the symplectic forms $\omega_{1}, \omega_{2}, \omega_{3}$ define a hyperkähler metric.

Proposition 4. $\phi$ is a Kähler potential with respect to the complex structure $J_{1}$.

Proof. From (12) and the discussion in section 2, it is clear that $z_{j}=x_{i}+i y_{j}$ for $1 \leq j \leq 2 n$ are complex coordinates in this complex structure. But $\phi$ is independent of $y_{j}$ so

$$
\partial \bar{\partial} \phi=\sum \frac{\partial^{2} \phi}{\partial x_{j} \partial x_{k}} d z_{j} \wedge d \bar{z}_{k}=-2 i \omega_{1}
$$

from (11).

REMARK. Note that the projection from $M \times \mathbf{R}^{2 n}$ to $M$ is holomorphic in the complex structure $J_{2}$. Using the $\partial, \bar{\partial}$ operators in that structure $\partial \bar{\partial} \phi$ gives the pullback of the Kähler form on $M$, which is degenerate.

Since $\omega_{j k}$ is constant and $\phi$ is independent of $y_{j}$, each symplectic form is invariant by the vector field $\partial / \partial y_{j}$. We thus have a triholomorphic action of $\mathbf{R}^{2 n}$ on $M \times \mathbf{R}^{2 n}$. The Legendre transform method is a canonical construction of hyperkähler manifolds $X^{4 n}$ with a triholomorphic action of $\mathbf{R}^{n}$, so in our case we have far more symmetry. 
Strictly speaking (a point not emphasized in the literature on this method) to apply the method we need an action which also admits an equivariant hyperkähler moment map. Recall that if $U$ is a triholomorphic vector field then for each $i, \iota(U) \omega_{i}=d \mu_{i}^{U}$ for some function $\mu_{i}^{U}$ and putting the moment maps $\mu_{i}^{U}$ together we get the hyperkähler moment map

$$
\mu: X \rightarrow \mathbf{R}^{n} \otimes \mathbf{R}^{3}
$$

which, if equivariant, represents $X$ as the total space of a principal $\mathbf{R}^{n}$ bundle over an open set of $\mathbf{R}^{n} \otimes \mathbf{R}^{3}$. The Legendre transform construction reduces the hyperkähler equations in this situation to finding a real-valued function $F\left(\mathbf{x}_{1}, \ldots, \mathbf{x}_{n}\right)$ defined on $\mathbf{R}^{n} \otimes \mathbf{R}^{3} \rightarrow \mathbf{R}$ and which satisfies the 3-dimensional Laplace equation

$$
\Delta F\left(c_{1} \mathbf{x}, \ldots, c_{n} \mathbf{x}\right)=0
$$

for each $\left(c_{1}, \ldots, c_{n}\right) \in \mathbf{R}^{n}$. Clearly if $F$ is defined from a holomorphic function $f\left(z_{1}, \ldots, z_{n}\right)$ by

$$
F\left(\mathbf{x}_{1}, \mathbf{x}_{2}, \ldots, \mathbf{x}_{n}\right)=\operatorname{Ref}\left(u_{1}+i v_{1}, \ldots, u_{n}+i v_{n}\right)
$$

with $\mathbf{x}_{j}=\left(u_{j}, v_{j}, w_{j}\right)$ then it satisfies this equation. In fact this is essentially the only way to obtain a solution which is invariant under translation in one of the coordinates of each $\mathbf{x}_{j} \in \mathbf{R}^{3}$.

Proposition 5. The hyperkähler metric defined by (11),(12) is constructed by the Legendre transform method from $F=R e \mathcal{F}$ where $\mathcal{F}$ is a holomorphic prepotential.

Proof. Recall that the introduction of the holomorphic function $\mathcal{F}$ required the choice of a symplectic basis on $V$. We shall need the sarne to implement the Legendre transform method, because we need an equivariant moment map. To see this note that if $U_{j}=-\partial / \partial y_{j}$, then from $(11),(12)$ the hyperkähler moment map is

$$
\begin{aligned}
\mu_{1} & =\frac{\partial \phi}{\partial x_{j}} \\
\mu_{2}+i \mu_{3} & =i \sum \omega_{j k}\left(x_{k}+i y_{k}\right)
\end{aligned}
$$

and this (because of the $y_{k}$ terms) is not equivariant for the full group $\mathbf{R}^{2 n}$ of isometries. However, if we choose a symplectic basis so that $\omega=\sum_{1}^{n} d x_{i} \wedge d x_{n+i}$ and take the action of $\mathbf{R}^{n}$ generated by $U_{1}, \ldots, U_{n}$, we have for $1 \leq j \leq n$ the moment map for $U_{j}$

$$
\begin{gathered}
\mu_{1}=\frac{\partial \phi}{\partial x_{j}}=\xi_{j} \\
\mu_{2}+i \mu_{3}=-i\left(x_{n+j}+i y_{n+j}\right)
\end{gathered}
$$

and this is equivariant since

$$
U_{j} \cdot y_{n+k}=-\frac{\partial y_{n+k}}{\partial y_{j}}=0
$$

for $1 \leq j \leq n$. The hyperkähler moment map is now:

$$
\mu(x)=\left(\xi_{1}, \ldots, \xi_{n},-y_{n+1}, \ldots,-y_{2 n}, x_{n+1}, \ldots, x_{2 n}\right) \in \mathbf{R}^{n} \otimes \mathbf{R}^{3}
$$


Since $\mathcal{F}$ is a holomorphic function of $w_{j}=\xi_{j}+i x_{n+j}, F=R e \mathcal{F}$ satisfies the equation (13).

To find the hyperkähler metric for such an $F$, we follow [7] putting

$$
z_{j}=-y_{n+j}+i x_{n+j}=i\left(x_{n+j}+i y_{n+j}\right)
$$

for $1 \leq j \leq n$. These are holomorphic functions with respect to the complex structure $J_{1}$. According to [7], a Kähler potential for this complex structure is

$$
K=F-\sum_{1}^{n}\left(u_{k}+\bar{u}_{k}\right) \xi_{k}
$$

where for $1 \leq j \leq n$

$$
\frac{\partial F}{\partial \xi_{j}}=u_{j}+\bar{u}_{j}
$$

But from (10) this means that $u_{j}+\bar{u}_{j}=x_{j}$ and

$$
K=F-\sum_{1}^{n} x_{k} \xi_{k}
$$

To summarize, we have $F\left(x_{n+1}, \ldots, x_{2 n}, \xi_{1}, \ldots, \xi_{n}\right)$ and from the complex Lagrangian submanifold structure of $M$ we obtain from (10) for $1 \leq j \leq n$,

$$
x_{j}=\frac{\partial F}{\partial \xi_{j}}, \quad \xi_{n+j}=-\frac{\partial F}{\partial x_{n+j}}
$$

From the $\Omega_{1}$-Lagrangian description of $M$ we also have, for $1 \leq j \leq 2 n$,

$$
\xi_{j}=\frac{\partial \phi}{\partial x_{j}}
$$

so in particular we can write (14) as

$$
K=F-\sum_{1}^{n} x_{k} \frac{\partial \phi}{\partial x_{k}}
$$

Differentiating with respect to $x_{j}$ for $1 \leq j \leq n$ we have

$$
\begin{aligned}
\frac{\partial K}{\partial x_{j}} & =\sum_{1}^{n} \frac{\partial F}{\partial \xi_{k}} \frac{\partial \xi_{k}}{\partial x_{j}}-\frac{\partial \phi}{\partial x_{j}}-\sum_{1}^{n} x_{k} \frac{\partial^{2} \phi}{\partial x_{k} \partial x_{j}} \\
& =\sum_{1}^{n} x_{k} \frac{\partial^{2} \phi}{\partial x_{k} \partial x_{j}}-\frac{\partial \phi}{\partial x_{j}}-\sum_{1}^{n} x_{k} \frac{\partial^{2} \phi}{\partial x_{k} \partial x_{j}} \\
& =-\frac{\partial \phi}{\partial x_{j}}
\end{aligned}
$$

using (15) and (16) and the fact that $F$ is independent of $x_{j}$ for $1 \leq j \leq n$. Similarly for $n+1 \leq j \leq 2 n$,

$$
\frac{\partial K}{\partial x_{j}}=\sum_{1}^{n} \frac{\partial F}{\partial \xi_{k}} \frac{\partial \xi_{k}}{\partial x_{j}}+\frac{\partial F}{\partial x_{j}}-\sum_{1}^{n} x_{k} \frac{\partial^{2} \phi}{\partial x_{k} \partial x_{j}}
$$




$$
\begin{aligned}
& =\sum_{1}^{n} x_{k} \frac{\partial^{2} \phi}{\partial x_{k} \partial x_{j}}-\xi_{j}-\sum_{1}^{n} x_{k} \frac{\partial^{2} \phi}{\partial x_{k} \partial x_{j}} \\
& =-\frac{\partial \phi}{\partial x_{j}}
\end{aligned}
$$

Hence $K=-\phi$ modulo an additive constant. Since $\phi$ is a Kähler potential from Proposition 5, we have the same metric (taking into account a difference of sign convention).

\section{REMARKS.}

1. To globalise this metric presents some choice. One could, as in [6], define it on $T^{*} M$. Its local structure is, however, that of a principal bundle with structure group a translation group. As such it has no geometrically distinguished zero section. In the context of complex Lagrangian submanifolds, it can be defined on the space of pairs of a complex Lagrangian submanifold together with a line bundle of fixed Chern class over it. In this context it is defined on an open set of Markman's moduli space [4] of Lagrangian sheaves, which is itself an integrable system.

2. In [8] a Kähler metric on the moduli space of pairs consisting of a special Lagrangian submanifold of a Calabi-Yau manifold together with a flat line bundle was defined and conjectured to be itself Calabi-Yau. In the case that the Calabi-Yau is hyperkähler and the special Lagrangian submanifold is complex Lagrangian with respect to one of the complex structures, this metric is precisely the one defined above. Since it is hyperkähler it is a fortiori Calabi-Yau.

3. From the point of view put forward in this paper we have travelled from a complex Lagrangian submanifold of $\mathbf{C}^{2 n}$ (Remark 1 of Section 4) to a hyperkähler metric. This is essentially the route followed by Cortes in [3].

\section{REFERENCES}

[1] M. F. Atryah And N. J. Hitchin, The geometry and dynamics of magnetic monopoles, M. B. Porter Lectures, Princeton University Press, Princeton, NJ, 1988.

[2] S. Cecotti, S. Ferrara and L. Girardello, Geometry of type II superstrings and the moduli space of superconformal theories, Int. J. Mod. Phys., A4 (1989), pp. 2475-2529.

[3] V. CoRTeS, On Hyper Kähler manifolds associated to Lagrangean Kähler submanifolds of $T^{*} \mathbf{C}^{n}$, Trans. Amer. Math. Soc., 350 (1998), pp. 3193-3205.

[4] R. Donagi And E. Markman, Spectral covers, algebraically completely integrable Hamiltonian systems and moduli of bundles, in Integrable Systems and Quantum Groups (Montecatini Terme, 1993), Lecture Notes in Mathematics, 1620, Springer, Berlin, 1996, pp. 1-119.

[5] R. Donagi, Seiberg-Witten integrable systems, Proc. Sympos. Pure Math., 62, Part 2, Amer. Math. Soc., Providence, RI, 1997.

[6] D. FreEd, Special Kähler manifolds, hep-th/9712042.

[7] N. J. Hitchin, A. Karlhede, U. Lindström, AND M. RočEK, Hyperkähler metrics and supersymmetry, Comm. Math. Phys., 108 (1987), pp. 535-589.

[8] N. J. Hitchin, The moduli space of Special Lagrangian submanifolds, Annali Scuola Normale Superiore di Pisa, (to appear) dg-ga/9711002.

[9] U.LindSTRÖM AND M.RoČEK, Scalar tensor duality and $N==1,2$ nonlinear $\sigma$ models, Nuclear Phys., B 222 (1983), pp. 285-308.

[10] ZHIQIN LU, A note on special Kähler manifolds, Columbia University preprint (1998).

[11] R. C. MCLean, Deformations of calibrated submanifolds, Comm. Anal. Geom., 6 (1997), pp. 705-747.

[12] C. Simpson, Higgs bundles and local systems, Inst. Hautes Études Sci. Publ. Math., 75 (1992), pp. 5-95.

[13] N. Seiberg AND E. Witten, Monopoles, duality and chiral symmetry breaking in $N=2$ supersymmetric QCD, Nuclear Phys., B431 (1994), pp. 484-550. 
[14] A. Strominger, S.-T. Yau, And E. Zaslow, Mirror symmetry is T-duality, Nuclear Phys., B479 (1996), pp. 243-259.

[15] C. Vorsin, Sur la stabilité des sous-variétés lagrangiennes des variétés symplectiques holomorphes, in Complex projective geometry (Trieste 1989/Bergen 1989), London Math. Soc. Lecture Notes, 179, Cambridge Univ. Press, Cambridge, 1992, pp. 294-303. 
N. J. HITCHIN 